\title{
La polisomnografía en noche dividida es eficaz para titular la presión en la ventilación con presión positiva continua ("CPAP") en pacientes con apnea obstructiva del sueño
}

\author{
Jorge Jorquera A ${ }^{1,2}$, Julia Santín 2,3 , Jaime G odoy 2,3. \\ Split night polysomnography \\ to titrate continuous positive airway \\ pressure therapy in adult patients \\ with obstructive sleep apnea
}

Background: Nasal continuous positive airway pressure therapy (CPAP) in the treatment of choice for adult obstructive sleep apnea (OSA). The diagnosis is established with polysomnography, but this study is expensive and must be repeated in those patients that require CPAP, to titrate the pressure of the therapy. Split polysomnography during one night to establish the diagnosis and titrate the pressure has been proposed to reduce costs. Aim: To assess if CPAP pressure can be adequately titrated in patients with OSA using a split-night polysomnography. Material and methods: One hundred fifty six patients with OSA were studied with split night polysomnography. CPAP pressure titration was considered adequate when there were less than five apnea/hypopnea episodes per hour, the registry time was more than 30 min, REM sleep occurred in more than $15 \%$ of the time and measurements were made in supine position. Results: An adequate titration was achieved in $80 \%$ of patients. The variables associated with an adequate titration were a higher registry time during the titration period, a higher percentage of stage III/IV or REM sleep during such period and the comfort experienced by the patient during the study. On the other hand, patients with an inadequate titration had a longer basal registry period. Conclusions: An adequate CPAP pressure can be prescribed to $80 \%$ of patients subjected to a split-night polysomnography. The basal registry period should not be longer than three hours, to allow an adequate titration lapse (Rev Méd Chile 2006; 134: 1377-83).

(Key words: Continous positive airway pressure; Polysomnography; Sleep apnea, obstructive)

Recibido el 28 de febrero, 2006. Aceptado el 27 de abril, 2006.

${ }^{1}$ Departamento de Enfermedades Respiratorias, ${ }^{2}$ Centro del Sueño, ${ }^{3}$ Departamento de Neurología, Pontificia Universidad Católica de Chile, Santiago de Chile.

Correspondencia a: Dr. Jorge Jorquera A. Departamento de Enfermedades Respiratorias y Centro del Sueño, Pontificia Universidad Católica de Chile. Marcoleta 352, Santiago. Teléfono: 02-6331541. Fax: 02-6335255. E mail: jjorquer@med.puc.cl 
$\mathrm{E}^{1}$ síndrome de apnea e hipopnea obstructiva del sueño (SAHOS), es una enfermedad que afecta a $4 \%$ de la población adulta ${ }^{1}$. Su síntoma cardinal es la somnolencia diurna excesiva ${ }^{2}$, que junto a la alteración del ánimo y deterioro cogniti$\mathrm{vo}^{3-5}$, producen un deterioro progresivo de la calidad de vida de los pacientes ${ }^{5-7}$. Además, se ha asociado a mayor riesgo de hipertensión arterial $^{8-10}$, morbimortalidad cardiovascular ${ }^{11}$, como también mayor riesgo de accidentes laborales y del tránsito ${ }^{12-14}$. El tratamiento de elección es la ventilación con presión positiva continua a través de una máscara nasal mientras duerme $(\mathrm{CPAPn})^{15}$.

La polisomnografía (PSG) basal es considerada el método de elección para establecer el diagnóstico del síndrome ${ }^{16}$, pero tiene el inconveniente de ser un estudio de alto costo, baja disponibilidad y requerir médicos altamente entrenados. Además, en pacientes que requieren tratamiento con CPAPn, se necesita un segundo estudio para establecer la presión necesaria para evitar las apneas, hipopneas y el ronquido. Con el propósito de reducir costos, en la evaluación de los pacientes con sospecha de SAHOS, se ha propuesto realizar el estudio PSG en dos etapas durante la misma noche, lo que se denomina PSG noche dividida ${ }^{17}$. Esta consiste en realizar una PSG estándar en la primera parte de la noche para realizar el diagnóstico, mientras que la segunda parte se establece la presión necesaria de CPAPn para el tratamiento. Algunos autores rechazan el estudio de PSG en noche dividida, argumentando que por el escaso tiempo de registro, la gravedad del SAHOS sería minimizada y que no sería posible titular adecuadamente a los pacientes ${ }^{18}$.

El objetivo de este trabajo fue evaluar la utilidad de la PSG noche dividida para establecer la presión adecuada en pacientes con alta sospecha de SAHOS e intención de tratamiento con CPAP.

\section{Pacientes y MÉTOdo}

Entre enero y agosto de 2002 se estudiaron, utilizando polisomnografías noche dividida (PSG ND), a 156 pacientes que consultaron por un cuadro sugerente de SAHOS, en el Centro del Sueño de nuestra institución. El estudio se realizó con un polisomnógrafo ALICE 3 Healthdyne, Technologies, siguiendo las normas de la Sociedad Americana de Trastornos del Sueño (ASDA) ${ }^{13}$. En todos los pacientes se monitorizaron: a) variables neurofisiológicas (electroencefalograma con ocho canales, electrooculograma y electromiograma del mentón), b) variables respiratorias (flujo nasal mediante cánula nasal y termistor, movimientos toracoabdominales y saturación de oxígeno $\left(\mathrm{SpO}_{2}\right)$ con un oxímetro de pulso digital). Además se registró electrocardiograma, posición corporal, ronquido mediante un micrófono y los movimientos de las extremidades inferiores mediante electrodos en la cara anterior de las piernas.

Antes de realizar la PSG, se aplicó una encuesta de síntomas, con el propósito de evaluar la presencia y magnitud de síntomas de SAHOS, que incluía entre otras la escala de somnolencia de Epworth. Antes de iniciar el estudio, se habituó a los pacientes al uso del CPAP, probándose distintos tipos y tamaños de máscaras nasales. Durante el estudio diagnóstico de la PSG ND, el registro fue evaluado continuamente por un técnico entrenado, que registró el número de apneas e hipopneas en cada hora durante las primeras dos o tres horas del estudio, para iniciar la titulación de la presión necesaria para el tratamiento con CPAPn. La decisión de realizar la titulación se basa en las normas de la ASDA ${ }^{19}$, que establece que se puede iniciar la titulación si durante la primera parte del estudio se observan más de 40 apneas o hipopneas por hora 0 se presentan entre 20 y 40 eventos respiratorios por hora, asociado a caída significativa de la saturación de oxígeno. Para la titulación con CPAP se utilizó un equipo REMstar ${ }^{\circledR}$, iniciando el estudio con $4 \mathrm{~cm} \mathrm{H}_{2} \mathrm{O}$ y aumentando gradualmente $1 \mathrm{~cm} \mathrm{H}_{2} \mathrm{O}$ hasta que los ronquidos, apneas, hipopneas y los eventos respiratorios relacionados con microdespertares fueron abolidos. Al finalizar la PSG se le solicitó al paciente calificar su experiencia con el dispositivo de CPAP como buena, regular o mala.

El registro PSG fue analizado manualmente por un médico entrenado, quien realizó la etapificación de las diferentes etapas del sueño, el conteo definitivo de los eventos respiratorios y los microdespertares, la evaluación del ronquido y de los movimientos de extremidades inferiores, tanto del registro basal como del obtenido con CPAPn. El análisis del estudio se realizó de acuerdo a las 
normas de la ASDA empleando las siguientes definiciones $^{16}$. a) Apnea obstructiva: ausencia del flujo oro nasal en presencia de movimientos tóraco-abdominales por más de 10 segundos de duración; b) Hipopneas: reducción del flujo aéreo en más de $50 \%$ por un período superior a 10 segundos asociado a caída de la $\mathrm{SpO}_{2}$ de 3\% o presencia de microdespertares; c) Microdespertar: aparición de onda alfa de 3 y 15 segundos de duración ${ }^{20}$. El índice de apnea hipopnea (IA/H), se determinó dividiendo el número total de eventos respiratorios por el tiempo total de sueño en horas.

Para considerar una titulación como adecuada con la presión elegida, se debían cumplir los siguientes criterios: 1) índice de apnea e hipopnea menor a 5

\section{Tabla 1. C aracterísticas clínicas y polisomnográficas de los pacientes estudiados}

\begin{tabular}{|lr|}
\hline $\mathrm{N}$ & 156 \\
Edad (años) & $52 \pm 11,8$ \\
Sexo (hombre) & $92 \%$ \\
Peso (K) & $89 \pm 16$ \\
Talla (cm) & $1,72 \pm 0,08$ \\
IMC (K/m²) & $30,1 \pm 5,1$ \\
Escala somnolencia Epworth & $13,7 \pm 5$ \\
Indice A/H (ev/h) & $52,3 \pm 25$ \\
Indice microdespertares (ev/h) & $53,1 \pm 22,8$ \\
SAHOS leve (IA/H 5-15) & $7,0 \%$ \\
SAHOS moderada (IA/H 15-30) & $14,7 \%$ \\
SAHOS grave (IA/H >30) & $78,2 \%$ \\
Saturación basal & $96,2 \pm 1,8$ \\
Saturación promedio & $86,6 \pm 5,6$ \\
Saturación mínima & $79,1 \pm 8,1$ \\
Ronquido (\% tiempo total sueño) & $24,2 \pm 16,5$ \\
Período latencia fase I (min.) & $12 \pm 17,3$ \\
\hline
\end{tabular}

eventos por hora, 2) tiempo del registro mayor de 30 minutos, 3) sueño REM mayor a $15 \%$ del tiempo total de sueño y 4) registro obtenido en posición supina.

Estadística. Los resultados fueron analizados mediante tests paramétricos (t-student) para el análisis de las variables continuas y test no paramétricos (chi cuadrado) en el análisis de las variables dicotómicas o categóricas. Se consideró estadísticamente significativo un valor de $\mathrm{p}<0,05$.

\section{RESULTADOS}

La Tabla 1 resume las características antropométricas de los pacientes. En $80 \%$ de los pacientes $(125 / 156)$ se pudo determinar adecuadamente la presión necesaria para el tratamiento con CPAPn. En los 31 pacientes restantes hubo incumplimiento de más de un criterio, por lo que no se consideró la titulación como adecuada. En $72 \%$ de éstos (22 pacientes), el registro con la presión sugerida fue inferior a 30 minutos; 68\% (21 pacientes) no cumplía el criterio del IA/H menor de 5 eventos/hora; 34\% (10 pacientes) no hubo registro en posición supina con la presión elegida, y $31 \%$ (9 pacientes) tenía un porcentaje de sueño REM $<15 \%$ del tiempo total del sueño.

En el grupo total, el tiempo promedio de registro basal fue de 209 min con un peńodo de latencia para alcanzar la fase I del sueño No REM de $12 \mathrm{~min}$. La arquitectura del sueño estuvo alterada en todos los pacientes, observándose un aumento de las etapas superficiales del sueño No REM en desmedro de las etapas profundas y del sueño REM. El empleo de CPAPn mejoró significativamente la arquitectura del sueño (Tabla 2). La Tabla 3 muestra que no hubo diferencias en las caracteństicas antropométricas, las

\section{Tabla 2. Arquitectura del sueño: arquitectura del sueño durante el estudio basal y con C PAP. Etapa I: representa el porcentaje del tiempo total de sueño que permanece en etapa I.}

\begin{tabular}{|lccr|}
\hline & Estudio basal & Estudio con CPAP & $p$ \\
\hline Tiempo registro (min.) & $209 \pm 49$ & $278 \pm 49$ & \\
$\%$ tiempo total de sueño & & & \\
Etapa I & $29,6 \pm 17$ & $19,3 \pm 14$ & $<0,00001$ \\
Etapa II & $49,5 \pm 14$ & $40,6 \pm 14$ & $<0,00001$ \\
Etapa III/IV & $13,8 \pm 10$ & $17,8 \pm 10$ & $<0,001$ \\
REM & $9,5 \pm 7,5$ & $22,3 \pm 10,7$ & $<0,00001$ \\
\hline
\end{tabular}


Tabla 3. Variables que no influyeron en la titulación adecuada del CPAP nasal

\begin{tabular}{|lccc|}
\hline & Titulación adecuada & Titulación no adecuada & $p$ \\
\hline Hombres & $112 / 124$ & $31 / 32$ & \\
Edad (años) & $51,6 \pm 1$ & $53,8 \pm 2,3$ & 0,17 \\
Peso (k) & $88,5 \pm 1,5$ & $93,5 \pm 1,6$ & 0,07 \\
IMC (k/m ${ }^{2}$ Escala de Epworth & $30,0 \pm 0,5$ & $30,6 \pm 0,9$ & 0,26 \\
Eficiencia (\%) & $13,6 \pm 0,5$ & $14,6 \pm 0,8$ & 0,21 \\
I A/H & $84,5 \pm 1,3$ & $84,6 \pm 2,1$ & 0,47 \\
I microdespertares & $52,1 \pm 2,2$ & $53,5 \pm 4,6$ & 0,39 \\
\end{tabular}

Tabla 4. Variables que influyeron en la titulación adecuada del CPAP nasal

\begin{tabular}{|lccr|}
\hline & Titulación adecuada & Titulación no adecuada & $p$ \\
\hline Tiempo registro basal (min.) & $203 \pm 3$ & $231 \pm 14$ & 0,002 \\
Tiempo registro CPAP (min.) & $285 \pm 3$ & $265 \pm 11$ & 0,004 \\
Etapa I con CPAP (\%) & $16,5 \pm 0,8$ & $29 \pm 5$ & $<0,0001$ \\
Etapa III/IV con CPAP (\%) & $18,6 \pm 10$ & $14,6 \pm 9$ & 0,03 \\
Etapa REM con CPAP (\%) & $23,4 \pm 0,9$ & $17,6 \pm 2$ & 0,04 \\
Tolerancia & $12 / 124$ & $17 / 32$ & $<0,0001$ \\
\hline
\end{tabular}

variables polisomnográficas basales y el grado de somnolencia entre el grupo que se pudo titular adecuadamente y aquél en que no fue posible hacerlo (Tabla 3). Las variables que influyeron positivamente en una adecuada titulación fueron: el mayor tiempo de registro durante el peńodo de titulación y el mayor porcentaje del tiempo total del sueño en etapa III/IV y del sueño REM durante este mismo peńodo. También pesó el mayor confort referido por el paciente al finalizar el estudio (Tabla 4). Las variables que influyeron negativamente en una adecuada titulación fueron: el mayor tiempo durante el registro basal y el mayor porcentaje del tiempo total del sueño en etapa I durante la fase de titulación (Tabla 4).

\section{Discusión}

El presente estudio demuestra que $80 \%$ de los pacientes a los cuales se les realizó una PSG ND, se pudo prescribir la presión necesaria para el tratamiento con CPAP nasal, resultado que es similar a lo publicado ${ }^{21}$ por otras series. Además, se demuestra que la posibilidad de titular adecuadamente la presión necesaria para abolir las apneas dependen: del tiempo de registro basal y del tiempo de registro con CPAPn. Es así como el menor tiempo destinado a establecer el diagnóstico, permite disponer de mayor tiempo para realizar una adecuada titulación. Estos hallazgos concuerdan con los comunicados por Yamashiro ${ }^{22}$, quien observó que un mayor tiempo de titulación aumenta la probabilidad de definir satisfactoriamente la prescripción de presión para el CPAPn. El menor tiempo promedio de registro basal en nuestros pacientes (210 min) en comparación a lo comunicado por otros autores, puede explicar el elevado porcentaje de éxito para establecer la presión adecuada en la presente serie.

Es posible que los diferentes resultados obtenidos al comparar la eficacia de la PSG ND con el sistema convencional se deba a los diferentes criterios utilizados para establecer la presión de titulación adecuada, considerada por Iber et $\mathrm{al}^{21}$ como la presión que disminuye el índice de eventos respiratorios a menos de 20 por hora, en cambio otros investigadores, no especifican los criterios empleados con este propósito ${ }^{17}$.

Una limitación del estudio es la imposibilidad de comparación del rendimiento obtenido con estudios en población semejante realizados en dos noches 
diferentes (estudio basal diagnóstico una noche y la segunda noche para titulación), aunque los criterios utilizados para establecer si el nivel de presión era la adecuada no han sido evaluados, comesponden a los empleados en la práctica clínica habitual: A) registro en posición supina, posición en la cual se producen los eventos respiratonios de mayor gravedad, B) presencia del sueño REM, etapa del sueño que se asocia a una disfunción más grave de la vía aérea supenior ${ }^{23-25}$, C) un $\mathrm{IA} / \mathrm{H}$ menor de 5 eventos por hora, considerado como nivel de normalidad de un PSG y D) un registro mayor a 30 minutos en la presión elegida.

Otras variables que influyen positivamente en lograr la adecuada titulación, fue la tolerancia al CPAP y la adaptación del paciente a la máscara nasal. El menor tiempo durante la etapa I durante el peńodo de titulación de CPAPn se debe probablemente a que los pacientes pudieron adaptarse mejor al dispositivo y dormir menos tiempo en etapas superficiales del sueño. El mayor tiempo durante las etapas profundas no REM y del sueño REM guarda relación con una mejor calidad de sueño y más reparador. En los pacientes que refieren mayor confort y tolerancia del dispositivo durante la noche hay más probabilidades de lograr una adecuada titulación. Nuestros pacientes estuvieron en contacto con el dispositivo, se probaron diferentes máscaras y se habituaron al uso del CPAP antes del examen, lo que puede explicar la buena adaptación y tolerancia a éste observada en $93 \%$ de ellos.

Observamos una tendencia, sin significación estadística ( $p=0,07$ ), entre el peso y la capacidad de titular adecuadamente a un paciente, lo cual nos hace suponer que los pacientes más obesos podnían tener mayor dificultad para ser titulados en una sola noche, debido a que requieren mayor presión, no toleran el dispositivo y eventualmente requieren el uso del BiPAP para su adecuado control ${ }^{26}$. Es necesario recordar que no a todos los pacientes vamos a solicitar una PSG ND, la que está indicada en pacientes con alta sospecha clínica de SAHOS y que

\section{REFERENCIAS}

1. Young T, Palta M, Dempsey J, Skatrud J, Weber $S$, BADR S. The occurrence of sleep-disondered breathing among middle-aged adults. NEngl J Med 1993; exista intención de tratamiento con CPAPn. Ante la sospecha de otros diagnósticos diferenciales es preferible realizar una PSG estándar con el fin de obtener el diagnóstico adecuado.

Se ha argumentado que la PSG ND podría no ser adecuada para evaluar la gravedad de la enfermedad y que en los pacientes sometidos a esta modalidad tendrían una menor adherencia al equipo de CPAP. Publicaciones recientes han demostrado que la PSG ND no subestima la gravedad de la enfermedad 17,27 y la adherencia al tratamiento es igual a la de los pacientes titulados con el estudio convencional ${ }^{28}$, con las ventajas de un tiempo de diagnóstico menor y un menor costo del sistema de salud.

Aun cuando otros autores ${ }^{21}$ han señalado, desde hace más de 10 años, que la PSG noche dividida permite establecer la presión efectiva de tratamiento con CPAPn en porcentajes semejantes a lo que nosotros observamos, esta modalidad de estudio no se ha implementado rutinariamente en nuestro país, probablemente por desconocimiento de la eficacia de este tipo de estudio. Nuestros resultados confirman que la PSG noche dividida permite en una sola noche confirmar el diagnóstico y determinar el tratamiento en aquellos pacientes con sospecha clínica de SAHOS, evitando la realización de un segundo estudio con la consiguiente duplicación de costos.

En resumen, en países como el nuestro, donde los recursos son limitados y los centros de sueño son escasos, es necesario optimizar los estudios polisomnográficos con el fin de disminuir los costos de diagnóstico e iniciar precozmente el tratamiento de los pacientes. Consideramos que una manera adecuada es realizar una PSG ND. Nuestros resultados avalan que la PSG ND permite confirmar el diagnóstico de SAHOS y prescribir la presión necesaria para su tratamiento en $80 \%$ de los pacientes. Para obtener estos resultados el tiempo de registro basal en lo posible no debe exceder las 3 horas, con el fin de disponer de más tiempo para la titulación, y el paciente debe familiarizarse previamente con el dispositivo para aumentar su tolerancia a éste.

328: 1230-5.

2. Engleman HM, Kingshott RN, Martin SE, Douglas NJ. Cognitive function in the sleep apnea/hypopnea syndrome (SAHS). Sleep 2000; 23 Suppl 4: S102-8.

3. Cheshire K, Engleman H, Deary I, Shapiro C, Douglas N. Factors impairing daytime performan- 
ce in patients with sleep apnea/hypopnea syndrome. Arch Intern Med 1992; 152: 538-41.

4. Guileminault C, Van Der Hoed J, Mttier M. Clinical overview of the sleep apnea syndromes. En: Guilleminault C, Dement WC, eds. Sleep Apnea Syndromes. New York, Alan R. Liss, 1978; pp. 1-12.

5. Smith I, ShneERson J. Is the SF36 sensitive to sleep disruption? A study in subjects with sleep apnoea. J Sleep Res 1995; 4: 183-8.

6. Jenkinson C, Strading J, Peterson S. Comparison of three measures of quality of life outcome in the evaluation of continuous positive airways pressure therapy for sleep apnoea. J Sleep Res 1997; 6: 199-204.

7. Jorquera J, Pacheco M, Freire M, Díaz O. Síndrome de apnea e hipopnea obstructiva del sueño y calidad de vida. Rev Ch de Enfer Resp 2003; 19 (3).

8. Peppard PE, Young T, Palta M, Skatrud J. Prospective study of the association between sleepdisordered breathing and hypertension. $\mathrm{N}$ Engl J Med 2000; 342: 1378-84.

9. Nieto FJ, Young TB, Lind BK, Shahar E, Samet JM, REDLNE S ET AL. Association of sleep-disordered breathing, sleep apnea, and hypertension in a large community-based study. Sleep Heart Health Study. JAMA 2000; 283: 1829-36.

10. Peker Y, Hedner J, Norum J, Kraiczi H, Carison J. Increased incidence of cardiovascular Disease in Middle-aged Men with Obstructive Sleep Apnea: A 7-Year Follow-up. Am J Respir Crit Care Med 2002; 166: 159-65.

11. Marín JM, Carrizo SJ, Vicente E, Agusti AG. Longterm cardiovascular outcomes in men with obstructive sleep apnoea-hypopnoea with or without treatment with continuous positive airway pressure: an observational study. Lancet 2005; 365: 1046-53.

12. Findley L, Unverzagt M, Suratt P. Automobile accidents involving patients with obstructive sleep apnea. Am Rev Respir Dis 1988; 138: 337-40.

13. George C, Nickerson P, Hanly P, Milar T, Kryger M. Sleep apnea patients have more automobile accidents. Lancet 1987; 2: 447.

14. Teran-Santos J, JimÉnez-Gómez A, Cordero-Guevara J, and the Cooperative Group Burgos-Santander. The association between sleep apnea and the risk of traffic accidents. N Engl J Med 1999; 340: 847-51.

15. Loube D, Gay P, Strohl K, Pack A, White D, CoLop $\mathrm{N}$. Indications for positive airway pressure treatment of adult obstructive sleep apnea patients a consensus statement. Chest 1999; 115: 863-6.
16. Sleep-related breathing disorders in adults: recommendations for syndrome definition and measurement techniques in clinical research. The Report of an American Academy of Sleep Medicine Task Force. Sleep 1999; 22: 667-89.

17. Mcardie N, Grove A, Devereux G, Mackay-Brown L, MACKaY T, Douglas NJ. Split-night versus full-night studies for sleep apnoea/hypopnoea syndrome. Eur Respir J 2000; 15: 670-5.

18. Fanfula F, Patruno V, Bruschi C, Rampula C. Obstructive sleep apnoea syndrome: is the halfnight polysomnography»an adequate method for evaluating sleep profile and respiratory events? Eur Respir J 1997; 10: 1725-9.

19. Chesson AL et al. ASDA Standards of Practice Committee. Practice parameters for the indications for polysomnography and related procedures. Sleep 1997; 20: 406-22.

20. Bonnet M, Carley D, and the Atlas Task Force. ASDA report. EEG arousals: scoring rules and examples. Sleep 1992; 15: 173-84.

21. Iber C, O'Brien C, Schluter J, Davies S, Leatherman J, MaHowaLd M. Single night studies in obstructive sleep apnea. Sleep 1991; 14: 383-5.

22. YAMASHIRO Y, KRYGER M. CPAP titration for sleep apnea using a split-night protocol. Chest 1995; 107: 62-6.

23. Charbonneau M, Martin J, Olha A, Kimoff J, Levy R, Cosio M. Changes on obstructive sleep apnea characteristics through the night. Chest 1994; 106 : 1695-701.

24. Strollo P, Sanders M, Constantino J, Walsh S, Stiler R, Atwood C. Split-night studies for the diagnosis and treatment of sleep-disordered breathing. Sleep 1996; 19: S255-S259.

25. Findiey L, WiLhoit S, SuRRat P. Apnea duration and hypoxemia during REM sleep in patients with obstructive sleep apnea. Chest 1985; 87: 432-6.

26. Weitzenblum E, Chaouat A. Sleep and chronic obstructive pulmonary disease. Sleep Med Rew 2004; 8: 281-94.

27. Jorquera J, Freire M, PACHeCo M. La polisomnografía en noche dividida es un método adecuado para valorar la severidad de la apnea obstructiva del sueño. Rev Ch de Enfer Resp 2003; 19 (3).

28. Fleury B, Rakotonanharv D, Tehindrazanarivelo A, Hausser-Hauw C, LebEau B. Long-term compliance to continuous positive airway pressure therapy (nCPAP) set up during a split-night polysomnography. Sleep 1994; 17: 512-5. 